Alex SAndro Rolland SouzA ${ }^{1}$

LARISSA ROCHA DE ANDRADE ${ }^{2}$

FELIPE LOPES TORRES DA SIIVA ${ }^{3}$

Ana Neves Cavalcanti ${ }^{3}$

GláuCia VIRGína de Querroz Lns Guerra ${ }^{3}$

\title{
Desfechos maternos e perinatais em gestantes com líquido amniótico diminuído
}

\author{
Maternal and perinatal outcomes \\ in women with decreased amniotic fluid
}

Artigo Original

Palavras-chave

Oligo-hidrâmnio

Líquido aminiótico

Ultrassonografia pré-natal

Hipertensão

Ruptura prematura de membranas fetais

Keywords

Oligohydramnios Amniotic fluid

Prenatal ultrasonography

Hypertension

Fetal membranes premature rupture

\section{Resumo}

OBJETIVO: Determinar os desfechos maternos e perinatais em gestantes com o líquido amniótico diminuído segundo o índice de líquido amniótico (ILA). MÉTODOS: Realizou-se estudo de coorte com 176 pacientes admitidas na enfermaria de alto risco do Instituto de Medicina Integral Prof. Fernando Figueira (IMIP. O líquido amniótico foi mensurado pelo índice de líquido amniótico, sendo classificado como diminuído, quando entre 5, 1 e 7,9 cm; oligohidrâmnio moderado, entre 3,1 e 5,0 cm; e grave, quando menor ou igual a 3,0 cm. Para se determinar a diferença entre os três grupos das variáveis categóricas estudadas, foram utilizados o teste de chi-quadrado e exato de Fisher, quando pertinentes, e, para as variáveis numéricas, utilizou-se o teste de Mann Whitney, em um nível de significância de 5\%. RESULTADOS: As malformações fetais ocorreram mais frequentes quando o oligohidrâmnio foi grave, enquanto as síndromes hipertensivas foram associadas ao oligohidrâmnio moderado. Observou-se semelhança entre os três grupos em relação à rotura prematura das membranas e outras causas. $\bigcirc$ líquido amniótico reduzido foi encontrado com maior frequência quando a idade gestacional do diagnóstico foi $\geq 32^{a}$ semana. Em relação aos desfechos perinatais, a incidência de índice de Apgar $<7$ no $1^{\circ}$ e $5^{\circ}$ minuto do óbito perinatal, da icterícia neonatal e da hipoplasia pulmonar foi mais elevada na presença do oligohidrâmnio moderado a grave. CONCLUSÕES: As causas e os desfechos maternos e perinatais em gestantes com líquido amniótico reduzido varia em relação a sua classificação pelo ILA, estando o oligohidrâmnio grave associado aos desfechos perinatais adversos e às malformações fetais.

Abstract

PURPOSE: To determine maternal and perinatal outcomes in pregnant women with low amniotic fluid, according to the amniotic fluid index (AFI). METHODS: A cohort study conducted on 176 patients admitted to the high risk ward of Instituto de Medicina Integral Prof. Fernando Figueira (IMIP), in Recife, Pernambuco, Brazil. Amniotic fluid was measured by the amniotic fluid index, and classified as low when between 5.1 and $7.9 \mathrm{~cm}$, moderate oligohydramnios between 3.1 and $5.0 \mathrm{~cm}$, and severe oligohydramnios when less than or equal to $3.0 \mathrm{~cm}$. To determine the difference between the three groups of categorical variables studied the chi-square and Fisher exact tests were used, when applicable, and for the numerical variables the Mann-Whitney test was applied, with the level of significance set at 5\%. RESULTS: Fetal malformation more frequently occurred when oligohydramnios was severe. Hypertensive disorders, however, were associated with moderate oligohydramnios. There was similarity between the three groups in relation to premature rupture of membranes and other causes. Low amniotic fluid was more frequently diagnosed when tested at the gestational age of 32 weeks or earlier. Regarding the perinatal outcomes, the incidence of Apgar score $<7$ in the 1 st and 5th minutes, perinatal death, neonatal jaundice and pulmonary hypoplasia was higher when oligohydramnios was moderate to severe. CONCLUSIONS: Maternal and perinatal causes and outcomes in pregnant women with low amniotic fluid vary with respect to their AFI, severe oligohydramnios being associated with fetal malformation and other adverse perinatal outcomes.

Correspondência

Alex Sandro Rolland Souzo Rua dos Coethos, 300 - Boa Vista (EP: $50070-550$ Recife (PE), Brasil

Recebido 08/05/2013
Trabalho realizado no Setor de Medicina Fetal do Instituto de Medicina Integral Prof. Fernando Figueira - IMIP - Recife (PE), Brasil. 'Programa de Residência Médica em Medicina Fetal, Instituto de Medicina Integral Prof. Fernando Figueira - IMIP - Recife (PE), Brasil; Departamento Materno Infantil, Universidade Federal de Pernambuco - UFPE - Recife (PE), Brasil.

${ }^{2}$ Curso Acadêmico de Medicina, Faculdade Pernambucana de Saúde - Recife (PE), Brasil.

${ }^{3}$ Instituto de Medicina Integral Prof. Fernando Figueira - IMIP - Recife (PE), Brasil. 


\section{Introdução}

O oligohidrâmnio é definido como uma redução da quantidade de líquido amniótico para menos de $500 \mathrm{~mL}$ com uma frequência que varia de 0,5 a $5,5 \%$ das gestações $^{1,2}$. A fisiopatologia é baseada nas anormalidades de sua produção, reabsorção ou perda. As principais causas são a rotura prematura das membranas, anomalias congênitas, síndrome de má adaptação placentária e uso de drogas pela mãe, podendo ou não estar associada aos desfechos perinatais adversos ${ }^{1,3,4}$.

Diante do diagnóstico de oligohidrâmnia, a rotura prematura das membranas deve ser sempre excluída, uma vez que acomete 8 a $10 \%$ das gestações. No entanto, essa condição é, em geral, facilmente diagnosticada clinicamen$\mathrm{te}^{5}$. As anomalias do trato urinário contribuem com $1 \%$ das malformações fetais, sendo geralmente associadas ao oligohidrâmnio grave em 25 a $38 \%$ das malformações do sistema urinário. Evolui com prognóstico fetal reservado, quando a redução do líquido é importante, particularmente devido a hipoplasia pulmonar e consequente óbito perinatal ${ }^{3,6}$. Nas anomalias cromossômicas sem envolvimento do sistema geniturinário, o oligohidrâmnio está presente em 0,4 a $4,4 \%$ dos $\operatorname{casos}^{6,7}$.

A má adaptação placentária é outra importante causa do oligohidrâmnio, pois leva à hipoxemia fetal crônica, provocando redução da diurese fetal. Está geralmente relacionada à hipertensão arterial, restrição de crescimento intrauterino, pós-maturidade e tabagismo materno ${ }^{8,9}$. Algumas drogas podem estar envolvidas na etiologia da redução do volume do líquido amniótico. Os inibidores da prostaglandina sintetase, como os anti-inflamatórios não hormonais e a dipirona, e os inibidores da enzima conversora de angiotensina provocam redução do fluxo vascular renal fetal e consequente oligohidrâmnio ${ }^{10,11}$. Outra droga pouco estudada, mas que pode causar o oligohidrâmnio, é a nifedipina ${ }^{12}$.

O líquido amniótico reduzido pode se associar aos resultados perinatais desfavoráveis, tanto devido às causas como pelas suas consequências sobre o feto ${ }^{8,9,13,14}$. Quanto mais precoce se instala o oligohidrâmnio, mais grave é o prognóstico materno e fetal ${ }^{14}$. Na presença da rotura das membranas amnióticas, tanto o maior tempo de bolsa quanto a menor quantidade de líquido amniótico, encontram-se associados às maiores taxas de infecções maternas e perinatais ${ }^{15,16}$.

O diagnóstico do oligohidrâmnio deve ser feito por meio da ultrassonografia, utilizando as técnicas da avaliação subjetiva ou da mensuração do maior bolsão na cavidade amniótica ou pelo índice de líquido amniótico (ILA $)^{4,13,17}$. A literatura sugere que a medida do maior bolsão seja preferível ao ILA por diagnosticar com menos frequência o oligohidrâmnio e por apresentar menor taxa de induções do trabalho de parto, contudo, sem melhorar os resultados perinatais ${ }^{13}$. Apesar disso, muitos centros utilizam o ILA por ser uma técnica de fácil realização e padronização, com boa reprodutibilidade e melhor quantificação do volume, sendo um método adequado para pesquisas ${ }^{13,15-17}$.

Nos diversos estudos, o ILA é classificado segundo a gravidade em oligohidrâmnio leve/líquido diminuído, moderado e grave. Acreditamos que, para cada grupo específico, as principais causas de oligohidrâmnio e os resultados perinatais sejam diferentes, podendo ajudar no diagnóstico diferencial; porém não encontramos estudos na literatura pesquisada. Assim, o objetivo desse estudo foi comparar as causas de redução do líquido amniótico e os resultados maternos e perinatais de acordo com a classificação pelo índice de líquido amniótico.

\section{Métodos}

Realizou-se estudo observacional, retrospectivo e tipo coorte, com gestantes internadas na enfermaria de alto risco do IMIP, no período de janeiro de 2007 a dezembro de 2008, em Recife (PE), localizado ao nordeste do Brasil.

O protocolo de pesquisa foi submetido ao comitê de ética em pesquisa do IMIP (CEP n $\left.{ }^{\circ} 786-06 / 2007\right)$ tendo sido iniciado apenas após a sua aprovação. Por se tratar de um estudo retrospectivo, foi solicitado ao CEP dispensa do Termo de Consentimento Livre e Esclarecido.

Foram incluídas todas as gestantes com diagnóstico de oligohidrâmnio admitidas na enfermaria de gestação de alto risco, no período do estudo, com idade gestacional maior ou igual à $24^{\mathrm{a}}$ semana e/ou peso do concepto ao nascimento maior ou igual a $500 \mathrm{~g}$. Foram excluídas as gestantes e os recém-nascidos cujos prontuários médicos não foram encontrados.

O cálculo do tamanho da amostra foi realizado pelo o programa Statcalc do Epi-Info 2007, versão 3.4.3, prevendo-se uma frequência de oligohidrâmnio, em gestações com malformações congênitas de fetos diagnosticadas no pré-natal, de $17 \%^{3}$ e uma precisão relativa de $30 \%$. Obteve-se uma amostra de 147 gestantes para um nível de confiança de $90 \%$.

As gestantes foram identificadas por meio de livro de registro do setor de Medicina Fetal, responsável pela realização das ultrassonografias da enfermaria de alto risco do IMIP. Dessa forma, o prontuário foi localizado e o instrumento de coleta preenchido adequadamente.

As características da amostra estudadas foram a idade materna (anos), a escolaridade (anos completos), a paridade e o trabalho remunerado. Os desfechos obstétricos analisados foram as causas da redução do líquido amniótico, o ILA, idade gestacional no diagnóstico e no nascimento (semanas), vias de parto (normal, cesárea e instrumental), 
as indicações de cesariana, corioamnionite, infecções materna e puerperal e dias de internação hospitalar materno.

Os desfechos perinatais foram óbito, sexo do recémnascido (masculino ou feminino), peso do recém-nascido (gramas), escore de Apgar no $1^{\circ}$ e $5^{\circ}$ minutos, infecções congênitas, icterícia neonatal, síndrome de desconforto respiratório (SDR) e/ou taquipneia transitória do recémnascido (TTRN), hipoplasia pulmonar, sepse neonatal e dias de internação hospitalar neonatal.

As gestantes foram internadas na enfermaria por diversas indicações, incluindo a de redução do líquido amniótico. Após a internação, todas as gestantes foram submetidas a, pelo menos, uma ultrassonografia obstétrica. Foi usado um equipamento Samsung Medison, modelo Accuvix V20 Prestige com transdutor setorial de $5 \mathrm{MHz}$. A gestante permaneceu, durante o exame, em decúbito dorsal. Todos os exames ultrassonográficos foram realizados por médicos especialistas em medicina fetal. A ultrassonografia foi realizada como rotina da instituição para avaliação do bem-estar fetal e da morfologia fetal, na qual se inclui a mensuração do ILA, podendo o diagnóstico da redução do volume de líquido aminiótico ter sido realizado ou ratificado durante a internação.

O volume do líquido amniótico foi avaliado pelo ILA, técnica semiquantitativa, na qual quatro bolsões de líquido amniótico são delimitados, dividindo-se o abdome materno em quatro quadrantes, por meio de duas linhas imaginárias perpendiculares que se cruzam no nível da cicatriz umbilical, sendo uma delas posicionada sobre a linha negra ${ }^{17}$. O somatório dos quatro valores determinou o valor do índice. Esse método bem padronizado demonstrou ter boa reprodutibilidade e ser de fácil aprendizado ${ }^{17}$.

As participantes foram divididas em três grupos segundo o valor do ILA.Considerou-se líquido amniótico diminuído, quando o valor encontrou-se entre 5,1 e 7,9 cm; oligohidrâmnio moderado, entre 3,1 a 5,0 cm; e oligohidrâmnio grave, quando menor ou igual a $3,0 \mathrm{~cm}^{17}$.

Os dados foram coletados pelos pesquisadores, utilizando-se o prontuário médico do setor de Medicina Fetal, além dos prontuários obstétricos e da neonatologia. Todas as gestantes foram acompanhadas até o momento do parto; e os recém-nascidos, até a alta hospitalar, para confirmação ou não dos diagnósticos sugeridos antes do parto. Os prontuários dos recém-nascidos foram utilizados para confirmar ou ratificar os diagnósticos.

As causas obstétricas da redução do líquido amniótico foram definidas cuidadosamente pelos pesquisadores, conforme descrito nos prontuários médicos. Assim, todas as gestantes incluídas no estudo tiveram seus diagnósticos confirmados ou ratificados retrospectivamente.

O diagnóstico de rotura prematura das membranas foi realizado por meio do exame especular quando se observou a saída de líquido amniótico pelo orifício cervical externo do colo uterino, durante a manobra de vassalva. As síndromes hipertensivas foram definidas segundo os critérios diagnósticos do National High Blood Pressure Education Program Working Group on High Blood Pressure in Pregnancy ${ }^{18}$. As doenças maternas associadas foram definidas segundo a informação da paciente ou quando o diagnóstico foi realizado durante a sua internação, de acordo com a descrição nos prontuários médicos. Consideraram-se causas idiopáticas, por exclusão diagnóstica, quando não foi evidenciada nenhuma possível etiologia da oligohidrâmnia descrita na literatura.

Para a confirmação das anomalias internas e das infecções congênitas diagnosticadas no pré-natal, foram consideradas as descrições dos exames complementares e/ou avaliação clínica e cirúrgica dos recém-nascidos. Para as anomalias externas, considerou-se o exame clínico realizado pelo neonatologista. Para avaliação do uso de drogas/medicações, foram observadas as prescrições médicas e relatórios de enfermagem, anotando aquelas que são possíveis causas de redução do volume de líquido amniótico.

Os desfechos obstétricos e perinatais também foram definidos por avaliação retrospectiva dos prontuários médicos, cujos diagnósticos foram realizados pelos médicos assistentes. Todos os diagnósticos foram revisados detalhadamente pelos pesquisadores, a fim de diminuir possíveis erros.

A análise estatística foi realizada com uso do programa de domínio público Epi-Info 3.5.3. Para descrever as características da amostra, utilizaram-se medidas de tendência central e dispersão, além de distribuições de frequências. Para comparação entre os grupos, utilizaram-se os testes de $\chi^{2}$ e exato de Fisher, quando pertinentes. As variáveis numéricas de distribuição assimétrica foram comparadas utilizando o teste de Mann Whitney. Todos os testes foram avaliados em um nível de significância de 5\%.

\section{Resultados}

Foram identificadas 219 gestantes, com diagnóstico ultrassonográfico de líquido amniótico reduzido ou oligohidrâmnio, candidatas à inclusão no estudo a partir da realização de, pelo menos, uma ultrassonografia obstétrica no setor de Medicina Fetal do IMIP. Dessas, 50 não foram incluídas na análise por ausência de registro neonatal, restando 176 gestantes para análise. Segundo o índice de líquido amniótico, 63 gestantes foram classificadas em líquido diminuído, 40 em moderado e 73 em grave.

De acordo com as principais características da amostra, a idade materna variou entre 13 e 43 anos com média de 25,6 6 6,9 anos. Quanto ao local de trabalhos, 96 pacientes $(55 \%)$ se declararam como trabalhadoras do lar; a maior parte, 65 gestantes $(37 \%)$, apresentava 
entre 8 e 11 anos completos de estudo; e 75 (43\%) eram primigestas. Destaca-se que não encontramos diferenças significativas quanto a essas características da amostra entre os três grupos (Tabela 1).

Quanto à causa básica da redução do índice de líquido amniótico, observou-se que para 54,0\% ( $\mathrm{n}=95)$ das participantes a redução foi devida a doenças clínicas e hipertensivas durante a gravidez e $41,5 \%(n=73)$ por rotura prematura das membranas; $26,1 \%(n=46)$ por malformações fetais; $4 \%(\mathrm{n}=7)$ por outras causas como infecções maternas e sífilis; e $8,5 \%(n=15)$ permaneceram como causas desconhecidas. Como possíveis drogas/medicações responsáveis pela redução do volume amniótico, a nifedipina foi a única descrita, sendo o seu uso observado em $15,9 \%(\mathrm{n}=28)$ das pacientes.

Observou-se ainda semelhança entre os grupos quanto à rotura prematura das membranas, doenças maternas associadas (incluindo diabetes); uso de nifedipina durante a internação; outras causas (qualquer infecção materna pré-natal ou congênita) e causas idiopáticas. As malformações fetais, de uma forma geral, ocorreram

Tabela 1. Características da amostra de acordo com a classificação do volume de líquido amniótico determinada pelo índice de líquido amniótico

\begin{tabular}{|c|c|c|c|c|}
\hline Variáveis & $\begin{array}{l}\text { Líquido } \\
\text { diminuido } \\
(n=63)\end{array}$ & $\begin{array}{l}\text { Oligohidrâmnio } \\
\text { moderado } \\
(n=40)\end{array}$ & $\begin{array}{l}\text { Oligohidrâmnio } \\
\text { grave (n=73) }\end{array}$ & Valor $p$ \\
\hline $\begin{array}{l}\text { Idade materna em anos } \\
\text { (Média } \pm \text { DP) }\end{array}$ & $25,3 \pm 7,5$ & $25,7 \pm 6,1$ & $25,9 \pm 7,0$ & $0,8^{\star}$ \\
\hline Nuliparas (n; \%) & $34 ; 54,0$ & $21 ; 52,5$ & $39 ; 53,4$ & $0,9 * \star$ \\
\hline Escolaridade $\leq 7$ anos $(\mathrm{n} ; \%)$ & $21 ; 33,3$ & $15 ; 37,5$ & $33 ; 45,2$ & $0,3^{\star \star}$ \\
\hline Trabalho remunerado $(n ; \%)$ & $40 ; 63,5$ & $24 ; 60,0$ & $46 ; 63,0$ & $0,9 * \star$ \\
\hline
\end{tabular}

mais frequentemente quando o oligohidrâmnio foi grave $(\mathrm{p}=0,0001)$, não havendo diferença entre os grupos quando as malformações do sistema geniturinário foram excluídas $(\mathrm{p}=0,3)$. As síndromes hipertensivas foram mais frequentemente associadas ao oligohidrâmnio moderado $(\mathrm{p}=0,002)$ (Tabela 2).

A mediana do índice de líquido amniótico de toda amostra foi de $4,6 \mathrm{~cm}$ (intervalo interquartil - IIQ: 2,4-5,7). Encontrou-se que a mediana do ILA foi significativamente maior nas gestantes que desenvolveram síndrome hipertensiva $(4,8 \times 3,1 \mathrm{~cm})$ quando comparada à mediana de ILA de todas as outras causas $(p=0,004)$. Nas gestações cujos recém-nascidos apresentavam diagnóstico de qualquer malformação fetal $(1,6 \times 4,6 \mathrm{~cm} ; \mathrm{p}<0,0001)$ e, particularmente quando a malformação fetal localizava-se no sistema geniturinário $(0 \times 4,6 \mathrm{~cm} ; \mathrm{p}=0,001)$, a mediana do ILA foi menor quando comparado à mediana das gestantes que não apresentavam malformações congênitas. Em relação à presença ou à ausência de rotura prematura das membranas $(3,8 x 4,5 \mathrm{~cm} ; \mathrm{p}=0,8)$, de doenças clínicas maternas $(4,7 \times 4,0 \mathrm{~cm} ; \mathrm{p}=0,1)$, do uso da nifedipina $(4,0 \times 4,0 \mathrm{~cm} ; \mathrm{p}=0,7)$, de outras causas $(1,3 \times 4,0 \mathrm{~cm}$; $\mathrm{p}=0,07)$ e de causas idiopáticas $(5,3 \times 4,0 \mathrm{~cm} ; \mathrm{p}=0,05)$, as medianas do ILA foram semelhantes nos grupo com e sem os desfechos estudados, respectivamente.

Em relação aos desfechos obstétricos, não se observou diferença significativa segundo a classificação de redução do volume de líquido amniótico para idade gestacional da interrupção antes ou após a $35^{\mathrm{a}}$ semana de gravidez, a frequência de cesariana, a corioamnionite, a infecção puerperal e a internação hospitalar com duração maior que 20 dias. Observou-se que a redução do volume do líquido amniótico, quando a idade gestacional do diagnóstico foi maior ou igual a $32^{\mathrm{a}}$ semana, foi frequentemente classificado como líquido amniótico reduzido ( $\mathrm{p}=0,002)$ (Tabela 3).

Tabela 2. Causas da redução volume de líquido amniótico de acordo com a classificação determinada pelo índice de líquido amniótico

\begin{tabular}{|c|c|c|c|c|c|c|c|}
\hline \multirow[t]{2}{*}{ Causas* } & \multicolumn{2}{|c|}{$\begin{array}{l}\text { Líquido diminuído } \\
\qquad(\mathrm{n}=63)\end{array}$} & \multicolumn{2}{|c|}{$\begin{array}{l}\text { Oligohidrâmnio moderado } \\
\qquad(\mathrm{n}=40)\end{array}$} & \multicolumn{2}{|c|}{$\begin{array}{c}\text { Oligohidrâmnio grave } \\
(n=73)\end{array}$} & \multirow[t]{2}{*}{ Valor $\mathrm{p}^{* *}$} \\
\hline & n & $\%$ & n & $\%$ & n & $\%$ & \\
\hline RPM & 24 & 38,1 & 20 & 50,0 & 29 & 39,7 & 0,4 \\
\hline Doenças clínicas & 12 & 19,0 & 6 & 15,0 & 9 & 12,3 & 0,5 \\
\hline Qualquer anomalia fetal & 7 & 11,1 & 8 & 20,0 & 31 & 42,5 & 0,0001 \\
\hline Uso de nifedipina & 11 & 17,4 & 6 & 15,0 & 11 & 15,1 & 0,9 \\
\hline Outras & 1 & 1,6 & 1 & 2,5 & 5 & 6,8 & 0,2 \\
\hline Idiopática & 8 & 12,7 & 3 & 7,5 & 4 & 5,5 & 0,3 \\
\hline
\end{tabular}

Líquido diminuído: 5,1 a $7,9 \mathrm{~cm}$; Oligohidrâmnio moderado: 3,1 a $5 \mathrm{~cm}$; Oligohidrâmnio grave: menor ou igual a $3 \mathrm{~cm}$; RPM: rotura prematura das membranas; SGU: sistema geniturinário. ${ }^{*}$ Um participante pode apresentar uma ou mais causa. ${ }^{\star}{ }^{*}$ Teste de $\chi^{2}$. 
Tabela 3. Desfechos obstétricos e perinatais de acordo com a classificação determinada pelo índice de líquido amniótico

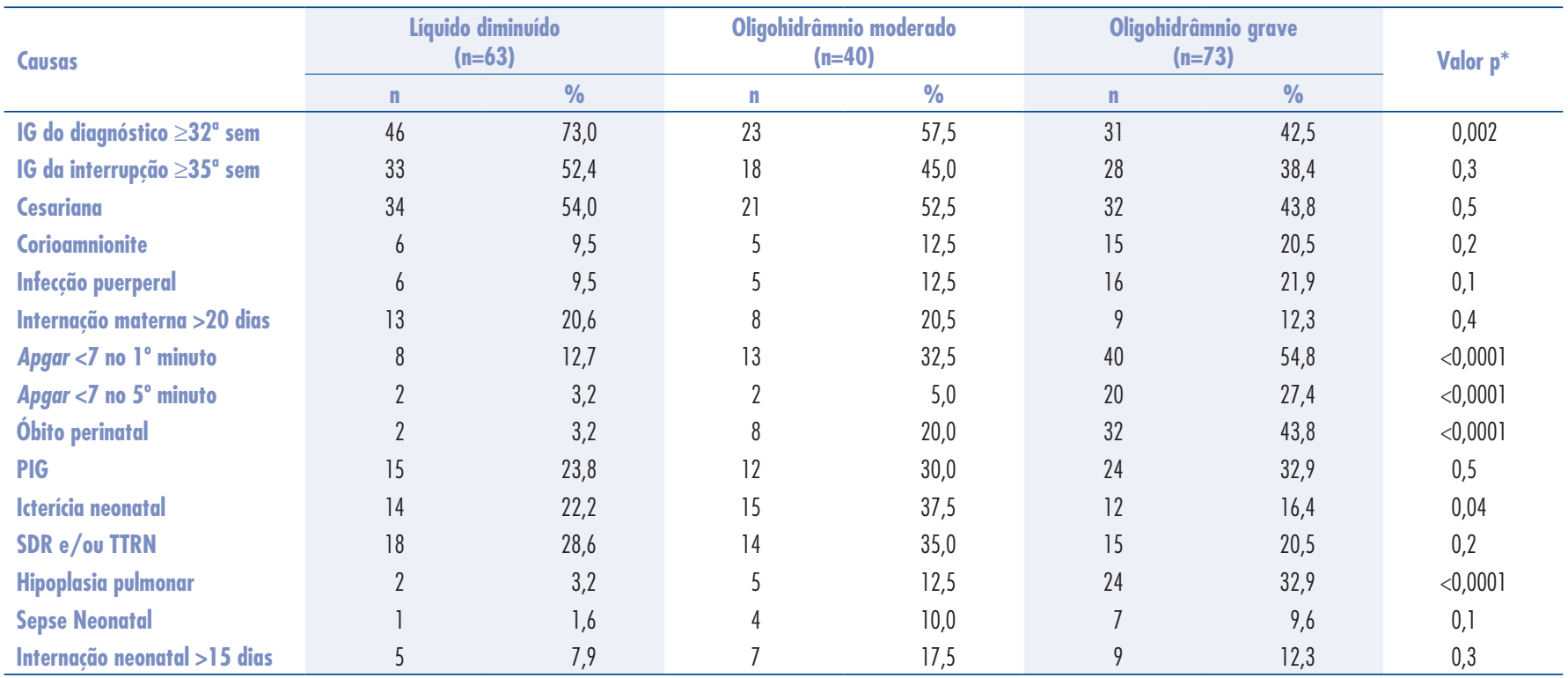

Líquido diminuído: 5,1 a $7,9 \mathrm{~cm}$; Oligohidrâmnio moderado: 3,1 a $5 \mathrm{~cm}$; Oligohidrâmnio grave: menor ou igual a $3 \mathrm{~cm}$; PIG: recém-nascido pequeno para idade gestacional; SDR: síndrome do desconforto respiratório do recém-nascido; TTRN: taquipnéia transitória do recém-nascido; IG: idade gestacional. *Teste de $\chi^{2}$.

As principais indicações de cesariana foram apresentações anômalas ( $\mathrm{n}=17-19,5 \%)$; oligohidrâmnio $(\mathrm{n}=16-18,4 \%)$; colo uterino desfavorável $(\mathrm{n}=14-16,1 \%)$, centralização fetal $(\mathrm{n}=11-12,6 \%)$, restrição de crescimento intrauterino $(\mathrm{n}=6-6,9 \%)$ e malformação fetal $(\mathrm{n}=5-5,7 \%)$.

Em relação aos desfechos perinatais, o índice de Apgar menor que sete no primeiro e no quinto minuto, o óbito perinatal, icterícia neonatal e a hipoplasia pulmonar foram mais frequentes na presença de oligohidrâmnio moderado a grave. Não se observou diferença significativa entre os grupos em relação aos recém-nascidos pequenos para idade gestacional, síndrome do desconforto respiratório do recémnascido ou taquipneia transitória do recém-nascido, sepse neonatal e dias de internação hospitalar maior que 15 dias.

\section{Discussão}

Neste estudo, as principais causas de oligohidrâmnio foram a rotura prematura das membranas, síndromes hipertensivas, doenças clínicas maternas associadas, malformações congênitas, o uso de nifedipina, infecções congênitas e causas idiopáticas semelhantes ao resultado em outros estudos 3 ,5-7,9-12,19.

A associação entre oligohidrâmnio e as síndromes hipertensivas e doenças clínicas maternas é bem documentada na literatura. Nessas situações, o oligohidrâmnio é devido a uma má adaptação placentária, o que representa maior gravidade da doença hipertensiva, contribuindo para elevar a morbidade e mortalidade perinatal, além de resultar em maiores taxas de indução do trabalho de parto ou indicação de cesariana ${ }^{9,20}$.
No nosso estudo, as síndromes hipertensivas foram mais frequentes quando o líquido amniótico era diminuído ou com oligohidrâmnio moderado. Em estudo com metodologia semelhante, foi encontrado que, na presença de restrição de crescimento intraútero, também decorrente da má adaptação placentária, o líquido amniótico, geralmente, encontra-se diminuído ou com oligohidrâmnio moderado ${ }^{21}$. Nesse estudo foi observado ainda maior frequência de hipertensão nas gestantes com ILA menor ou igual a $5 \mathrm{~cm}(11 \%)$, quando comparado ao grupo de ILA entre 5 e $8 \mathrm{~cm}(5 \%)^{21}$.

A rotura prematura das membranas foi observada em $41,5 \%$ das gestantes. Porém, uma revisão descreve que a rotura prematura das membranas acomete 8 a $17 \%$ de todas as gestações e 2 a $4 \%$ das gestações prematuras ${ }^{5,22}$. Essa frequência elevada em nossa amostra se deve a população estudada de gestantes com oligohidrâmnio. A rotura prematura das membranas é uma importante causa de líquido diminuído/oligohidrâmnio, a qual leva a graves repercussões maternas e perinatais, contribuindo para uma mortalidade perinatal de 18 a 20\% nos Estados Unidos da América ${ }^{5,16}$.

No nosso estudo, não foi demonstrado diferença significativa segundo a classificação do ILA, nem diferença na sua mediana. Destaca-se a constante renovação do líquido amniótico nessas situações, com sua produção pela urina e perda pela rotura. Em um estudo, observou-se que em gestantes com oligohidrâmnio o ILA permaneceu constante nas pacientes que não foram submetidas à hidratação oral ${ }^{23}$, corroborando com os nossos resultados de que o volume do líquido amniótico não tende a diminuir rapidamente até o ponto de chegar a aumentar a frequência de oligohidrâmnio grave. 
As malformações congênitas se encontram associadas às alterações do volume do líquido amniótico (oligohidrâmnio ou polihidrâmnio) $)^{3}$. Em nosso estudo, observou-se uma elevada frequência de fetos malformados, sendo $13 \%$ de malformação do sistema urinário, provavelmente pela instituição ser um centro de referência em Medicina Fetal.

Observou-se associação mais forte do oligohidrâmnio grave quando estavam presentes anomalias congênitas do sistema geniturinário. Porém não foi encontrada diferença significativa quando as malformações urinárias foram excluídas da análise, sugerindo que o sistema geniturinário seja o principal fator determinante desta associação. Estudo semelhante ao nosso observou que o ILA menor ou igual a $5 \mathrm{~cm}$ foi mais frequente na presença de malformações maiores $(25 \%)$ comparados ao grupo com ILA entre 5,1 e $8 \mathrm{~cm}$ ou superior a $8 \mathrm{~cm}^{21}$.

Na literatura, algumas drogas utilizadas durante a gestação encontram-se associadas ao líquido diminuído/oligohidrâmnio, como anti-inflamatórios não hormonais, inibidores da enzima conversora da angiotensina, drogas ilícitas, dentre outras $^{10-12}$. Nos últimos anos, a nifedipina vem sendo amplamente utilizada para inibição do trabalho de parto prematuro $^{12}$. No nosso estudo, observou-se uma frequência de líquido diminuído/oligohidrâmnio de $16 \%$, sem diferença significativa entre os grupos segundo a classificação do ILA. Essa diminuição provavelmente é por tempo limitado e sem causar repercussões perinatais, podendo ser justificada por alterações sobre a hemodinâmica materna e fetal, observada em estudo por meio da doplervelocimetria ${ }^{12}$.

A idade gestacional do diagnóstico após a $32^{\mathrm{a}}$ semana de líquido diminuído/oligohidrâmnio foi mais frequente quando o ILA encontrava-se entre 5 e $8 \mathrm{~cm}$. Em um estudo, observou-se que a idade gestacional média do diagnóstico de líquido diminuído menor ou igual a $5 \mathrm{~cm}$ foi na $34^{\mathrm{a}} \mathrm{se}-$ mana ${ }^{19}$. Destaca a diferença da população estudada que foi apenas em oligohidrâmnio isolado e sem causa determinada.

Em relação à idade gestacional do parto após a $35^{\mathrm{a}}$ semana, não foi observada diferença significativa entre os três grupos. Porém com uma frequência maior nos casos de oligohidrâmnio moderado e grave ${ }^{21}$. Isso pode sugerir uma diminuição lenta do líquido amniótico com o evoluir da gravidez. A interrupção da gestação frequentemente realizada em torno da $35^{\text {a }}$ semana pode ter sido o fator contribuinte para não se ter observado uma diferença significativa ${ }^{19}$.

Não existe consenso na literatura que defina um ponto de corte no índice de líquido amniótico que defina quando a cesariana eletiva é protetora para o feto, existindo um fraco grau de recomendação a favor da realização da indução do trabalho de parto quando o ILA for maior que $5 \mathrm{~cm}^{24,25}$. Ressaltamos que a indução do trabalho de parto está associada a uma maior chance de frequência cardíaca fetal não tranquilizadora, presença de mecônio, baixos escores de Apgar e maior chance de cesariana de urgência ${ }^{24,25}$. Todavia, os estudos não observaram diferenças importantes quanto aos desfechos perinatais ${ }^{24}$.
Apesar da taxa de cesariana ter isso alta em nosso estudo, observamos que o ILA não influenciou na frequência segundo a classificação, o que pode ter ocorrido por sermos um centro de referências com indicações restritas, além das diferentes causas de volume de líquido amniótico reduzido. Nos casos de rotura prematura das membranas, a cesariana é menos realizada, enquanto, nas síndromes hipertensivas, a cesariana é frequentemente indicada ${ }^{24-26}$.

Destacamos no nosso estudo que houve maior frequência de infecções puerperal e corioamnionite nas pacientes o oligohidrâmnio grave, apesar de não haver diferença significativa entre os grupos. Em relação ao recém-nascido, observou maior frequência de sepse neonatal na presença de oligohidrâmnio moderado/grave. Estudos sugerem que quanto menor o ILA, em pacientes com rotura prematura das membranas, maior a frequência de infecções maternas e perinatais ${ }^{19,27}$.

Em relação aos resultados perinatais, observamos maior frequência de $A$ pgar menor que sete no $1^{\circ}$ e no $5^{\circ}$ minuto, óbito perinatal e hipoplasia pulmonar na presença de oligohidrâmnio grave, semelhante ao estudo que comparou o oligohidrâmnio isolado com o de causa definida ${ }^{28}$.

A icterícia neonatal em nosso estudo foi mais frequente com o líquido diminuído ou oligohidrâmnio moderado, ao contrário de outro estudo no qual não se evidenciou diferença entre os grupos com e sem oligohidrâmnio ${ }^{19}$. Enquanto a síndrome do desconforto respiratório do recém-nascido (SDR) e/ou taquipneia transitória do recém-nascido (TTRN) não diferiu entre os três grupos estudados. É provável que o tamanho amostral tenha influenciado nesses resultados, mas uma possível justificativa para a frequência da icterícia neonatal, da SDR e da TTRN tenha sido associada à interrupção antecipada da gravidez.

Destacamos que nosso estudo foi retrospectivo, incluindo todas as causas de redução do volume amniótico, para podermos determinar o nosso objetivo principal, impossibilitando uma análise específica dos desfechos maternos e perinatais para as causas específicas. Outra limitação se depara sobre as diferentes metodologias, critérios de inclusão e número de sujeitos dos diversos estudos confrontados, além de não ter sido encontrado estudo semelhante ao nosso na literatura pesquisada.

Assim, concluímos que as causas e os desfechos maternos e perinatais em gestantes com líquido amniótico reduzido varia em relação a sua classificação pelo ILA, estando o oligohidrâmnio grave associado a desfechos perinatais adversos e a malformações fetais.

\section{Agradecimentos}

Ao Programa Institucional de Bolsas de Iniciação Científica (PIBIC) do Instituto de Medicina Integral Prof. Fernando Figueira e ao Conselho Nacional de Desenvolvimento Científico e Tecnológico (CNPq). 
1. ACOG Committee on Practice Bulletins-Obstetrics ACGO Practice Bulletin No. 80: premature rupture of membranes. Clinical management guidelines for obstetrician-gynecologists American College of Obstetricians and Gynecologists. Obstet Gynecol. 2007; 109(4): 1007-19.

2. Munn MB. Management of oligohydramnios in pregnancy. Obstet Gynecol Clin North Am. 201 1;38(2):387-95.

3. Neto CN, Souza AS, Moraes Filho OB, Noronha AM. [Amniotic fluid volume associated with fetal anomalies diagnosed in a reference center in the Brazilian Northeast]. Rev Bras Ginecol Obstet.2009;31(4):164-70. Portuguese.

4. Freire DM, Cecatti JG, Paiva CS. [Is uterine height able to diagnose amniotic fluid volume deviations?]. Rev Bras Ginecol Obstet. 2013;35(2):49-54. Portuguese.

5. Caughey AB, Robinson JN, Norwitz ER. Contemporary diagnosis and management of preterm premature rupture of membranes. Rev Obstet Gynecol. 2008;1(1):1 1-22.

6. John U, Benz K, Hübler A, Patzer L, Zenker M, Amann K. Oligohydramnios associated with sonographically normal kidneys. Urology. 2012;79(5): $1155-7$.

7. Kamath-Rayne BD, Saal H, Lang S, Habli M. Recurrent severe oligohydramnios and fetal pulmonary hypoplasia associated with ErbB4 mutation. Obstet Gynecol. 2013;121 (2 Pł 2 Suppl 1):499-501

8. Yuan W, Duffner AM, Chen L, Hunt LP, Sellers SM, Bernal AL. Analysis of preterm deliveries below 35 weeks' gestation in a tertiary referral hospital in the UK. A case-control survey. BMC Res Notes. 2010;3:119.

9. Parlakgumus HA, Iskender C, Aytac PC, Tarim E. Do intrauterine growth restricted fetuses of the hypertensive and normotensive mothers differ from each other?. Arch Gynecol Obstet. 2012;286(5): 1 147-51.

10. Shrim A, Berger H, Kingdom J, Hamoudi A, Shah PS, Koren G. Prolonged exposure to angiotensin-converting enzyme inhibitors during pregnancy. Fetal toxicity could be reversible Can Fam Physician. 2005;51(10):1535-7.

11. Weintraub A, Mankuta D. Dipyrone-induced oligohydramnios and ductus arteriosus restriction. Isr Med Assoc J. 2006;8(10):722-3.

12. Lima MM, Souza AS, Diniz C, Porto AM, Amorim MM, Moron AF. Doppler velocimetry of the uterine, umbilical and fetal middle cerebral arteries in pregnant women undergoing tocolysis with oral nifedipine. Ultrasound Obstet Gynecol. 2009;34(3):3 11 1-5.

13. Nabhan AF, Abdelmoula YA. Amniotic fluid index versus single deepest vertical pocket as a screening test for preventing adverse pregnancy outcome. Cochrane Database System Rev. 2008;(3):CD006593.

14. Peralta $C F$, Figueiredo Neto $M$, Hidalgo SR, Sbragia Neto $L$, Rosa IRM, Marussi EF, et al. [Fetal bilateral obstructive uropathies: ultrasound findings during pregnancy and postnatal outcomes]. Rev Bras Ginecol Obstet. 2009;31(1 1):540-6. Portuguese.

15. Volante E, Gramellini D, Moretti S, Kaihura C, Bevilacqua G. Alteration of the amniotic fluid and neonatal outcome. Acta Biomed. 2004;75(Suppl 1):71-5.

16. Test G, Levy A, Wiznitzer A, Mazor M, Holcberg G, Zlotnik A, Sheiner $\mathrm{E}$. Factors affecting the latency period in patients with preterm premature rupture of membranes. Arch Gynecol Obstet. $2011 ; 283(4): 707-10$.

17. Phelan JP, Smith CV, Broussard P, Small M. Amniotic fuid volume assessment with the four-quadrant technique at 36-42 weeks' gestation. J Reprod Med. 1987;32(8):540-2.

18. National High Blood Pressure Education Program Working Group on High Blood Pressure in Pregnancy. Report of the National High Blood Pressure Education Program Working Group on High Blood Pressure in Pregnancy. Am J Obstet Gynecol. 2000; 183(1):S1-S22.

19. Melamed N, Pardo J, Milstein R, Chen R, Hod M, Yogev Y. Perinatal outcome in pregnancies complicated by isolated oligohydramnios diagnosed before 37 weeks of gestation. Am J Obstet Gynecol. $2011 ; 205(3): 241$.e 1-6.

20. Noronha Neto C, Souza AS, Amorim MM. [Pre-eclampsia treatment according to scientific evidence]. Rev Bras Ginecol Obstet. 2010;32(9):459-68. Portuguese.

21. Petrozella LN, Dashe JS, McIntire DD, Leveno KJ. Clinical significance of borderline amniotic fluid index and oligohydramnios in preterm pregnancy. Obstet Gynecol. $2011 ; 117(2 \mathrm{Pt} 1): 338-42$.

22. Luton D, Alran S, Fourchotte V, Sibony $O$, Oury JF. Paris heat wave and oligohydramnios. Am J Obstet Gynecol. 2004;191 (6):2103-5.

23. Akter MD, Kabir N, Shah MS, Islam F, Tasnim S. Effect of maternal oral hydration therapy in oligohydramnios. Mymensingh Med J. 2012;21(4):723-8.

24. Mozurkewich E, Chilimigras J, Koepke E, Keeton K, King VJ. Indications for induction of labour: a best-evidence review. BJOG. 2009; 1 16(5):626-36.

25. Tucker EB, Fager C, Srinivas S, Lorch S. Predictors of cesarean delivery for periviable neonates. Obstet Gynecol. 2011 ; 1 18(1):4956. doi:10.1097/AOG.0b013e31821c4071

26. Pádua SK, Osis MD, Faúndes A, Barbosa AH, Moraes Filho $\mathrm{OB}$. Fatores associados à realização de cesariana em hospitais brasileiros. Rev Saúde Pública. 2010; 44(1):70-9.

27. Flenady $\mathrm{V}$, King JF. Antibiotics for prelabour rupture of membranes at or near term. Cochrane Database Syst Rev. 2002;(3):CD00 1807.

28. Zhang J, Troendle J, Meikle S, Klebanoffa MA, Rayburn WF. Isolated oligohydramnios is not associated with adverse perinatal outcomes. BJOG. 2004; 111 (3):220-5. 